\title{
Atypical Presentation of Gastric Volvulus
}

\author{
Denzil Etienne ${ }^{a}$, b Mel A. Ona ${ }^{a}$, Madhavi Reddy ${ }^{a}$
}

\begin{abstract}
Gastric volvulus has been reported in all age groups; however, it is typically diagnosed in the elderly. Organo-axial volvulus is the most common type, followed by the mesentero-axial and the combined types. Depending on the etiology, gastric volvulus can also be classified as primary (idiopathic) or secondary. Treatment of acute gastric volvulus involves laparoscopic or endoscopic procedures/maneuvers, depending on the severity of symptoms and surgical eligibility of the patient. Chronic gastric volvulus is typically managed conservatively, although laparoscopic or endoscopic interventions can also be employed depending on the severity of presentation.
\end{abstract}

Keywords: Volvulus; Gastric; Organo-axial

\section{Introduction}

Gastric volvulus has been reported in all age groups; however, it is typically diagnosed in the elderly. The range of clinical presentations can range from an incidental radiographic finding to life-threatening emergencies, depending on the rate of progression and extent of gastric rotation. Thus, gastric volvulus constitutes an important differential diagnosis in select patients presenting with abdominal pain and vomiting. The following case describes an incidental finding of gastric volvulus in an elderly patient with striking radiographic and endoscopic images. A discussion of the etiology, classification, clinical presentation and management of gastric volvulus follows.

\section{Case Report}

A 68-year-old female with a past medical history significant

Manuscript accepted for publication March 09, 2017

aDepartment of Gastroenterology and Hepatology, The Brooklyn Hospital Center, Academic Affiliate of The Icahn School of Medicine at Mount Sinai, Clinical Affiliate of The Mount Sinai Hospital, 121 Dekalb Avenue, Brooklyn, New York 11201, USA

${ }^{\mathrm{b}}$ Corresponding Author: Denzil Etienne, Department of Gastroenterology and Hepatology, The Brooklyn Hospital Center, Academic Affiliate of The Icahn School of Medicine at Mount Sinai, Clinical Affiliate of The Mount Sinai Hospital, 121 Dekalb Avenue, Brooklyn, NY 11201, USA.

Email: denziletienne@gmail.com

doi: https://doi.org/10.14740/gr813w for gastric ulcer presented with fever and was admitted for sepsis of unknown etiology. Physical examination was noncontributory. Laboratory results demonstrated normal white blood cell count and anemia: hemoglobin and hematocrit of $8.5 \mathrm{~g} / \mathrm{dL}$ and $27 \%$, respectively. Iron studies were indicative of iron deficiency anemia. The platelet count was 178,000 cells/L. Fecal occult blood test was positive. As part of the sepsis workup, a computed tomography (CT) scan of the abdomen subsequently revealed rotation of the stomach along the long axis, suggestive of organo-axial volvulus (Fig. 1). Upper gastrointestinal (GI) series demonstrated herniation of the entire stomach through the diaphragmatic esophageal hiatus (Fig. 2). The stomach was rotated upon itself (arrow in Fig.2), which corroborated the CT findings of organo-axial volvulus. No associated obstruction, ulceration, malrotation, or masses were identified. Subsequent esophagogastroduodenoscopy (EGD) revealed esophagitis in the distal third of the esophagus with spiral rotation of the gastric mucosal folds (Fig. 3), which confirmed the diagnosis of organo-axial volvulus. The patient was managed conservatively as her gastric volvulus was asymptomatic and likely represented an incidental chronic finding. The etiology of her fever was not discovered despite an extensive workup; however, the patient had prompt resolution of presenting symptoms after initiation of antibiotics and was discharged shortly afterwards.

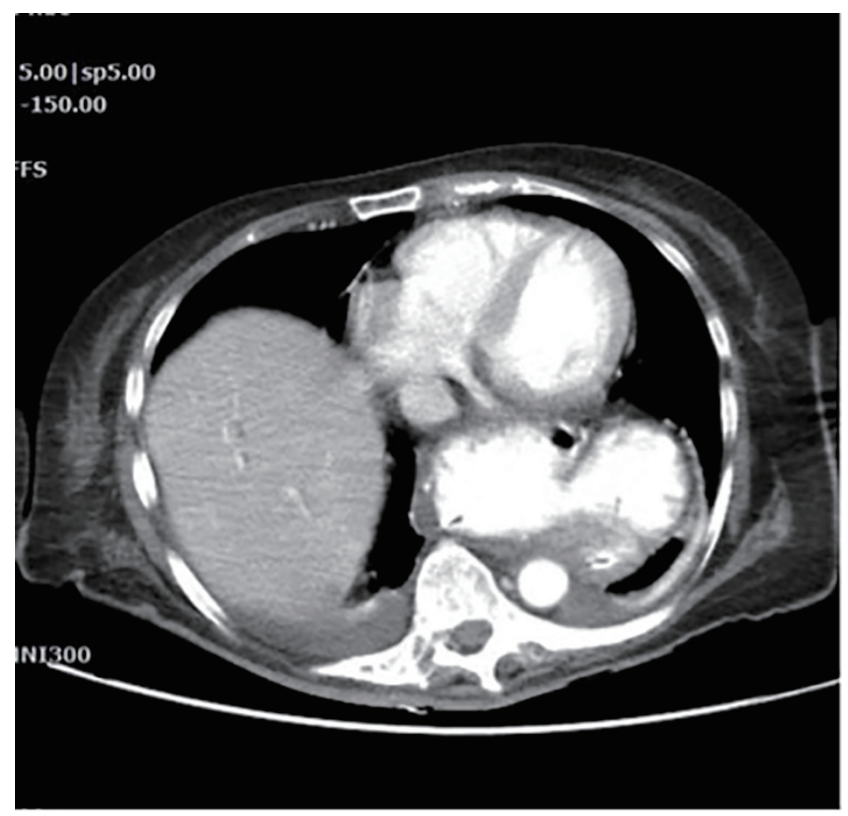

Figure 1. CT abdomen/pelvis showing organo-axial gastric volvulus. 


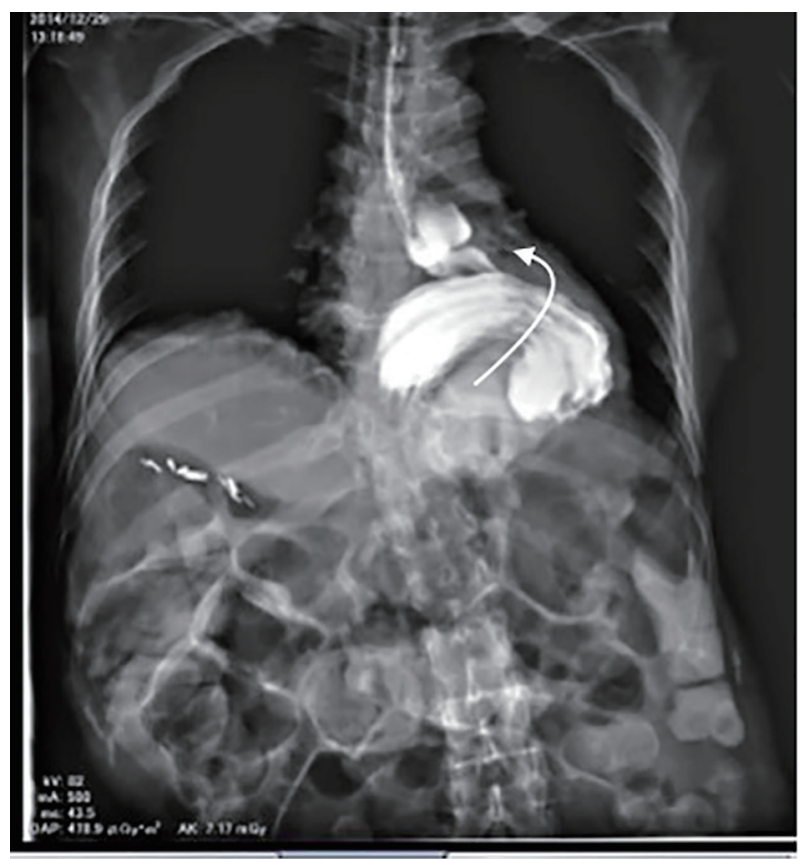

Figure 2. Upper GI series showing organo-axial volvulus (arrow depicting rotational direction).

\section{Discussion}

Volvulus rarely involves the stomach [1]. While gastric volvulus has been reported in all age groups, it is typically diagnosed in elderly patients $[1,2]$. There are three known types of gastric volvulus. In decreasing order of frequency, organo-axial volvulus is the most common, followed by the mesentero-axial type and lastly, the combined type [1]. Depending on the etiology, gastric volvulus can also be classified as primary (idiopathic) or secondary. Primary gastric volvulus occurs due to abnormalities of the gastric ligaments that lead to failed gastric fixation [3]. Secondary gastric volvulus arises in the presence of local anatomic abnormalities, including paraesophageal hernia, diaphragmatic hernia, phrenic nerve paralysis and atypical anatomy of adjacent organs [4]. The range of presentations can vary from incidental radiographic findings to life-threatening emergencies, depending on the rate of progression and extent of gastric rotation [3, 4]. Acutely, patients can present with chest pain, abdominal pain, and vomiting [3]. Abdominal distention, rigidity, and guarding can be noted on physical examination [5]. Confirmatory studies include X-ray and CT. Treatment of acute gastric volvulus involves laparoscopic and/ or endoscopic procedures/maneuvers, depending on the severity of symptoms and surgical eligibility of the patient. Chronic gastric volvulus can be managed conservatively or by laparoscopic and/or endoscopic interventions. The patient described above most likely represented a case of chronic gastric volvulus secondary to a widened esophageal hiatus with resultant reflux esophagitis. Among the most common complications of chronic volvulus are gastric ulceration and gastro-esophageal reflux with esophagitis [6], the latter of which may have led to iron deficiency anemia in our patient.

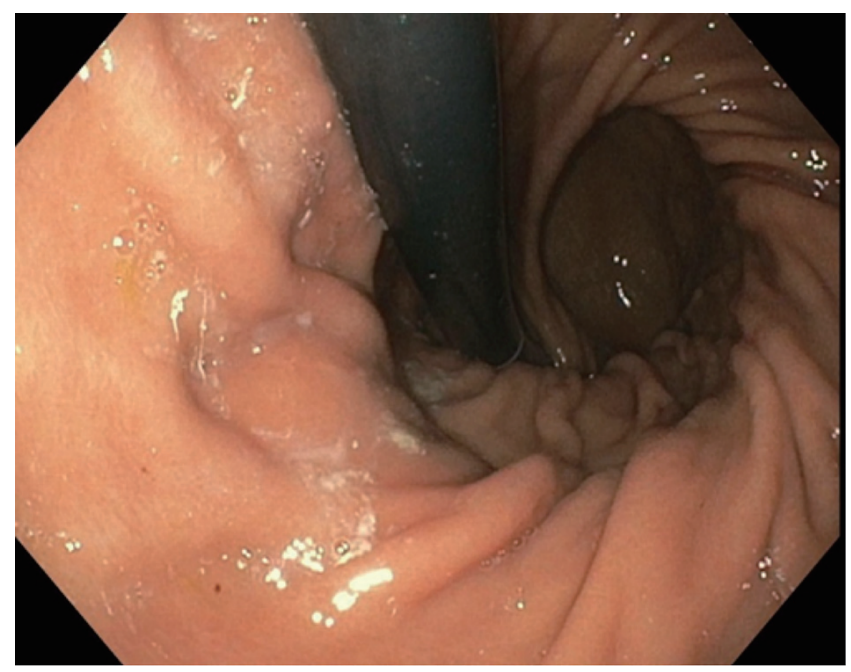

Figure 3. EGD showing spiral rotation of gastric mucosal folds upon retroflexion.

\section{Author Contributions}

Conception and design: Denzil Etienne, MD, Mel A. Ona, MD, and Madhavi Reddy, MD. Drafting of the article: Denzil Etienne, MD and Mel A. Ona, MD. Critical revision of the article for important intellectual content: Denzil Etienne, MD, Mel A. Ona, MD, and Madhavi Reddy, MD. Final approval of the article: Denzil Etienne, MD, Mel A. Ona, MD, and Madhavi Reddy, MD.

\section{Conflicts of Interest}

None of the authors have any conflicts of interest (financial or otherwise) to disclose.

\section{References}

1. Peterson CM, Anderson JS, Hara AK, Carenza JW, Menias CO. Volvulus of the gastrointestinal tract: appearances at multimodality imaging. Radiographics. 2009;29(5):1281-1293.

2. Wastell C, Ellis H. Volvulus of the stomach. A review with a report of 8 cases. Br J Surg. 1971;58(8):557-562.

3. Wasselle JA, Norman J. Acute gastric volvulus: pathogenesis, diagnosis, and treatment. Am J Gastroenterol. 1993;88(10):1780-1784.

4. Rashid F, Thangarajah T, Mulvey D, Larvin M, Iftikhar SY. A review article on gastric volvulus: a challenge to diagnosis and management. Int J Surg. 2010;8(1):18-24.

5. McElreath DP, Olden KW, Aduli F. Hiccups: a subtle sign in the clinical diagnosis of gastric volvulus and a review of the literature. Dig Dis Sci. 2008;53(11):3033-3036.

6. Patel NM. Chronic gastric volvulus: report of a case and review of literature. Am J Gastroenterol. 1985;80(3):170173. 